\title{
3D Indoor Localization using Visible Light Communications
}

\author{
Amirhosein Hajihoseini ${ }^{1}$, Akbar Dargahi ${ }^{2}$, Seyed Ali Ghorashi ${ }^{1,3}$ \\ Cognitive Telecommunication Research Group, Faculty of Electrical Engineering, \\ Shahid Beheshti University G. C., Tehran, $\operatorname{Iran}^{1}$ \\ Faculty of Electrical Engineering, Shahid Beheshti University G. C., Tehran, Iran ${ }^{2}$ \\ Cyber Space Research Institute, Shahid Beheshti University G. C., Tehran, Iran ${ }^{3}$
}

\begin{abstract}
Due to increasing demand for wireless spectrum and considering its scarcity, wireless optical communications technology is expected to be used in future systems. Recently, visible light communications (VLC) is introduced as an alternative to the global positioning system (GPS) to be used inside the buildings where GPS does not work properly. In this paper, a new method for localization of an optical receiver is proposed in which, information received from indoor optical transmitters is used. In this method, first the angle of arrival of received signal is calculated, using the field of view of optical transmitter and then least square estimation (LSE) is used to localize the receiver in 3 dimensional indoor environment. Simulation results show that we the location of optical receiver in 3D indoor environment can be estimated using 4 visual access pints with approximately 0.6 meters average error.
\end{abstract}

Keywords: Localization; Visible Light Communications; Least Square Estimator; Optical Communication.

\section{INTRODUCTION}

Increasing in usage of devices which use wireless In this paper, we estimate the location of VLC receiver in spectrum makes spectrum scarcity as a big challenge for 3 dimensional environment of a room using AOA method. new wireless devices. This forces researchers to find In the next sections we show that localizing target cannot alternative resources to solve this problem. Using optical be performed by an acceptable error using two visual band is an appropriate alternative to deal with spectrum access points (VAPs) which consist of some LEDs. scarcity [1]. This concept in communication systems is Simulation results show that the estimated location of known as topics such as free space optical (FSO) target has an acceptable error.

communications or optical wireless communication (OWC). In this system, transmitter transmits an optical In the rest of this paper, in section II we express beam and receiver convert this beam to the information. assumptions and introduce formulation of the problem. In The receiver is typically an optical diode. If this systems section III we explain the proposed algorithm and simulate use visible light band, the concept is known as visible light some scenarios to evaluate the performance of the communications (VLC).

Recently, VLC has attracts many researchers [2-4]. In this systems, light emitting diode (LED) is typically used as transmitter [5]. One of the applications of VLC is localization of devices in indoor places or each place which couldn't use global positioning system (GPS). Several methods exist to collect information from environment and analyze them to localize target [6]. Received signal strength (RSS), time of arrival (TOA), time difference of arrival (TDOA) and angle of arrival (AOA) are some methods which are used to localize target. proposed algorithm in section IV, and finally the paper in concluded in section $\mathrm{V}$.

\section{SYSTEM MODEL AND PROBLEM FORMULATION}

In this paper we use VLC systems to localize target in indoor places. We assume that there are some VAPs with arrays of LEDs. These transmitters have limited field of view (FOV) and due to this limitation, they can scan a limited range of angle. Figure 1 illustrates a VAP in the corner of a room.

We assume that the considered device for localization is a

Examples of localization using optical communication introduced in [7, 8]. In this paper, authors use TDOA to localize target in indoor places. This method unlike TOA method does not need synchronization of transmitter and receiver. Authors in [9] use RSS and AOA jointly to localize target by using optical communications. In [5] authors localize a VLC receiver in 2 dimensional indoor place using AOA.

VLC receiver. This receiver can connect to one of the VAPs in each time instance. Each LED transmits optical beam to the special angular range and we assume that transmitters of each VAP can scan its environment, completely. Also we put a special code to the message of each LED, therefore, the receiver can know by using this code that it is connected to which LED and this LED belongs to which VAP. 
INTERNATIONAL JOURNAL OF INNOVATIVE RESEARCH IN ELECTRICAL, ELECTRONICS, INSTRUMENTATION AND CONTROL ENGINEERING Vol. 4, Issue 7, July 2016

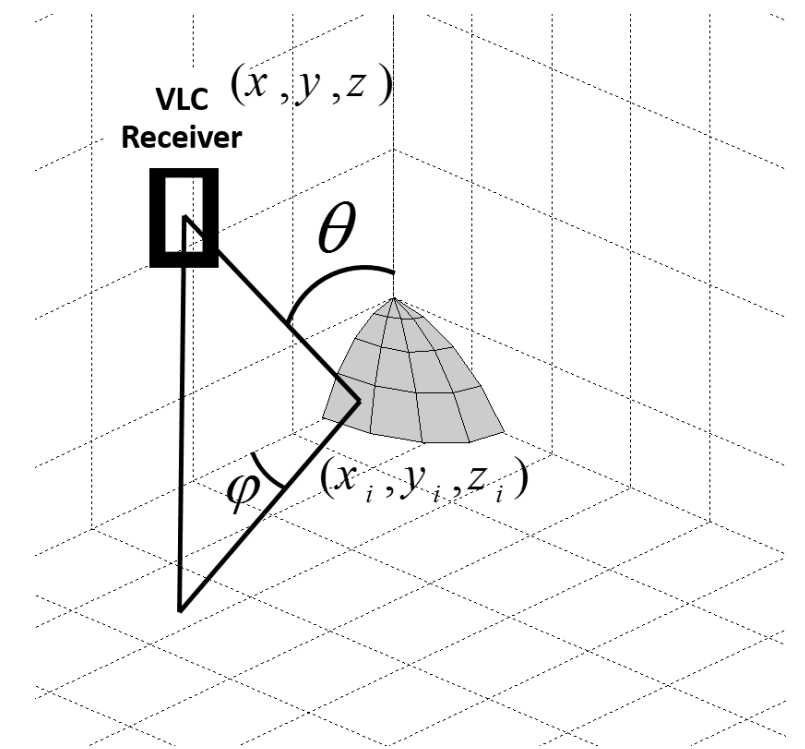

Fig. 1 Topology of the considered scenario

Also we assume that the receiver knows the location of each VAP. Therefore, receiver can estimate its angle to each VAP by connecting to LEDs.

\section{INDOOR VLC LOCALIZATION}

In this paper, similar to [5] the localization problem is solved by using connection of receiver information and VAPs, however, in this paper we extend the algorithm for 3D places. By using connections' information, receiver measures the azimuthal and polar angle to each VAP and by using these angles, it can determine that receiver is in the FOV of which LED and can localize itself.

If receiver is connected to two VAPs, and detects that connection is done with which LEDs of each VAP, it can localize itself. However, if there are more than two VAPs, the arrived angles become more, and receiver estimates its location using least square estimator (LSE).

We assume that there are some VAPs in the room. We use $\varphi$ and $\theta$ as azimuthal and polar angles between receiver and each VAP, $[\mathrm{x}, \mathrm{y}, \mathrm{z}]$ as the location of VLC receiver and $\left[\mathrm{x}_{\mathrm{i}}, \mathrm{y}_{\mathrm{i}}, \mathrm{z}_{\mathrm{i}}\right]$ as a location of the $\mathrm{i}^{\text {th }} \mathrm{VAP}$. Therefore we have:

$$
\begin{gathered}
\tan \phi_{i}=\frac{y-y_{i}}{x-x_{i}} \\
\tan \theta_{i}=\frac{z-z_{i}}{\sqrt{\left(x-x_{i}\right)^{2}+\left(y-y_{i}\right)^{2}}}
\end{gathered}
$$

By expanding (1) we have:

$$
x \sin \varphi_{i}-y \cos \varphi_{i}=x_{i} \sin \varphi_{i}-y_{i} \cos \varphi_{i}
$$

Now we assume that we have $\mathrm{N}$ VAPs and rewrite formula in matrix form as (4),

$$
A x=b
$$

where

$$
A=\left[\begin{array}{cc}
\sin \varphi_{1} & -\cos \varphi_{1} \\
\sin \varphi_{2} & -\cos \varphi_{2} \\
\vdots & \vdots \\
\sin \varphi_{N} & -\cos \varphi_{N}
\end{array}\right] \text { and } b=\left[\begin{array}{c}
x_{1} \sin \varphi_{1}-y_{1} \cos \varphi_{1} \\
x_{2} \sin \varphi_{2}-y_{2} \cos \varphi_{2} \\
\vdots \\
x_{N} \sin \varphi_{N}-y_{N} \cos \varphi_{N}
\end{array}\right]
$$

Then $[\mathrm{x}, \mathrm{y}]$ is calculated using $\operatorname{LSE}(5)$.

$$
[x, y]=\left(A^{T} A\right)^{-1} A^{T} b
$$

Now we want to calculate the height of VLC receiver. For this, we extend (2) and rewrite it in matrix form. The height of VLC receiver is calculated using (6) to (8).

$$
\begin{gathered}
z \cos \theta_{i}=z_{i} \cos \theta_{i}+\sin \theta_{i} \sqrt{\left(x-x_{i}\right)^{2}+\left(y-y_{i}\right)^{2}} \\
C=\left[\begin{array}{c}
\cos \theta_{1} \\
\cos \theta_{2} \\
\vdots \\
\cos \theta_{N}
\end{array}\right], d=\left[\begin{array}{c}
\sin \theta_{1} \sqrt{\left(x-x_{1}\right)^{2}+\left(y-y_{1}\right)^{2}} \\
\sin \theta_{2} \sqrt{\left(x-x_{2}\right)^{2}+\left(y-y_{2}\right)^{2}} \\
\vdots \\
\sin \theta_{N} \sqrt{\left(x-x_{N}\right)^{2}+\left(y-y_{N}\right)^{2}}
\end{array}\right] \\
z=\left(C^{T} C\right)^{-1} C^{T} d
\end{gathered}
$$

\section{SIMULATION RESULTS}

In this section, we perform some scenarios to evaluate the performance of VLC localization. We assume that a VLC receiver exists in a $4 \times 6 \times 5[\mathrm{~m}]$ room. In this room there are 4 VAPs where locations of them are depicted in Table 1. In each scenario we investigate 4 cases depicted in Table 2 .

\section{TABLE I LOCATION OF VAPS}

\begin{tabular}{|c|c|c|c|c|}
\hline & VAP 1 & VAP 2 & VAP 3 & VAP 4 \\
\hline $\mathrm{x}[\mathrm{m}]$ & 0 & 0 & 4 & 4 \\
\hline $\mathrm{y}[\mathrm{m}]$ & 0 & 6 & 6 & 0 \\
\hline $\mathrm{z}[\mathrm{m}]$ & 0 & 5 & 5 & 0 \\
\hline
\end{tabular}

TABLE II 4 CASES FOR VAPS

\begin{tabular}{|c|c|}
\hline Case A & $\begin{array}{c}\text { All VAPs are active and measure polar and } \\
\text { azimuthal angle }\end{array}$ \\
\hline Case B & $\begin{array}{c}\text { VAP 1 and VAP 2 are active and measure } \\
\text { polar and azimuthal angle }\end{array}$ \\
\hline Case C & $\begin{array}{c}\text { VAP 3 and VAP 4 are active and measure } \\
\text { polar and azimuthal angle }\end{array}$ \\
\hline Case D & $\begin{array}{c}\text { VAP 1 and VAP 2 measure polar angle and } \\
\text { VAP 3 and VAP 4 measure azimuthal angle }\end{array}$ \\
\hline
\end{tabular}

A. VLC Localization

In first scenario, we assume each VAP has $4 \mathrm{x} 4$ LED array and move VLC receiver in whole places of room in the height of 2.5 meter and try to localize it. Figures 2 to 5 illustrate the RMSE for 4 cases. As depicted in these 
INTERNATIONAL JOURNAL OF INNOVATIVE RESEARCH IN ELECTRICAL, ELECTRONICS, INSTRUMENTATION AND CONTROL ENGINEERING Vol. 4, Issue 7, July 2016

figures, by using VAPs we can localize VLC receiver with error less than 0.5 meter.

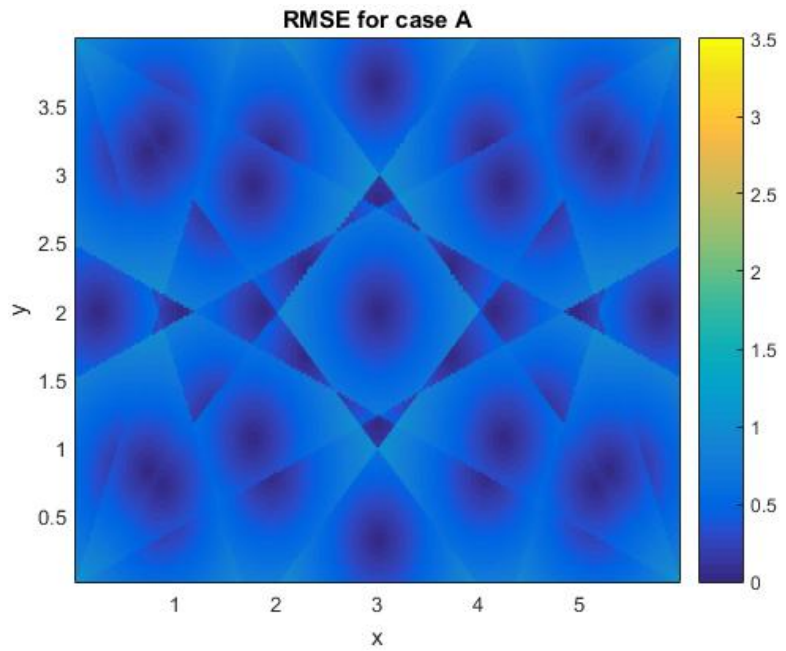

Fig. 2 RMSE of VLC localization in case A.

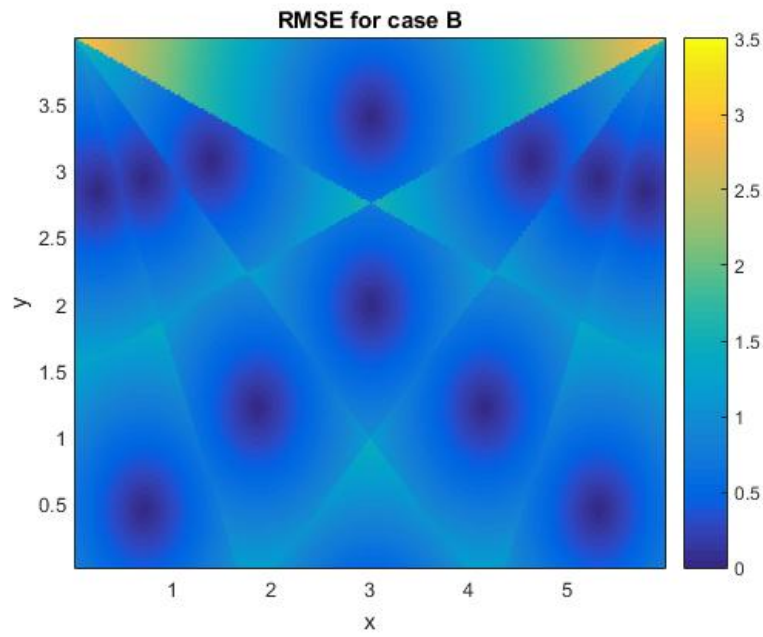

Fig. 3 RMSE of VLC localization in case B.

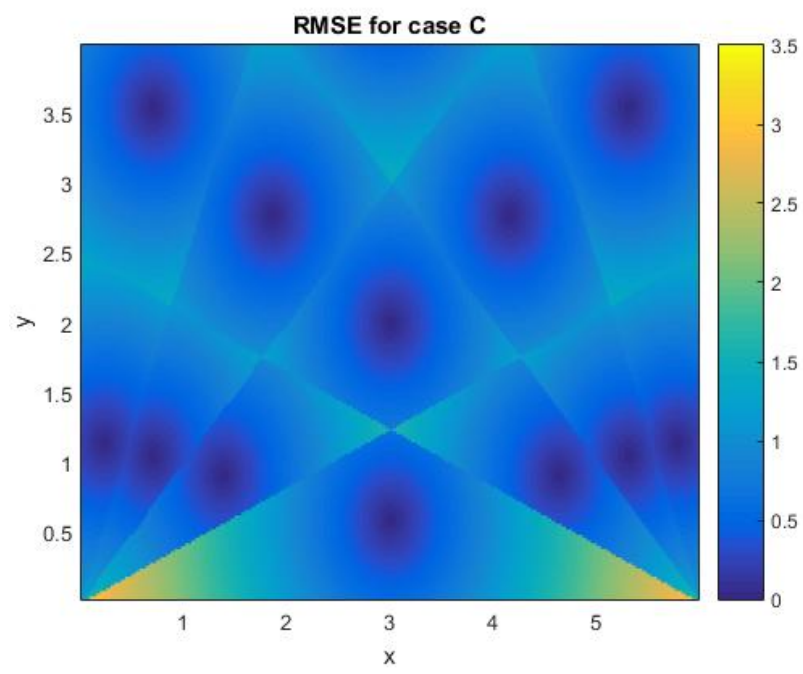

Fig. 4 RMSE of VLC localization in case C.

According to activeness of all VAPs in case A, in this case (Figure 2) the location of VLC receiver is estimated by an acceptable error in all places of room.

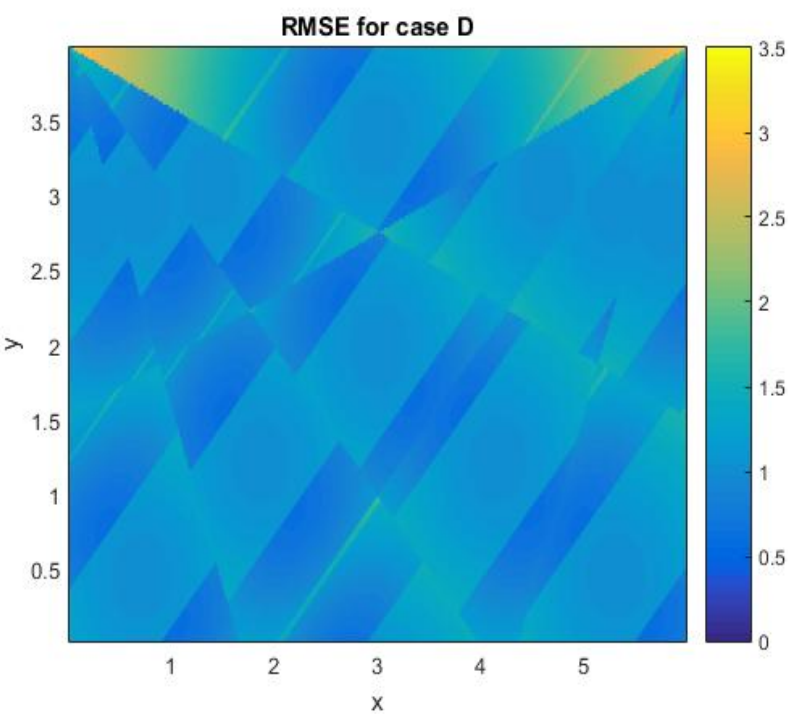

Fig. 5 RMSE of VLC localization in case D.

In cases B and C (Figure 3 and 4), due to activeness of two VAPs, error becomes more than what is in case A, however, error is still acceptable. In Figure 5 error is almost the same as cases $\mathrm{B}$ and $\mathrm{C}$, but the complexity of case $\mathrm{D}$ is less than other.

\section{B. Effect of Height of VLC Receiver}

In this scenario, we investigate the effect of VLC height in the performance of localization. Therefore, we move receiver in all places of room and calculate the RMSE of localization. Then, we average the RMSE at each height. In this scenario, we assume that each VAP has an array of 5x5 LED. Result are depicted in Figure 6.

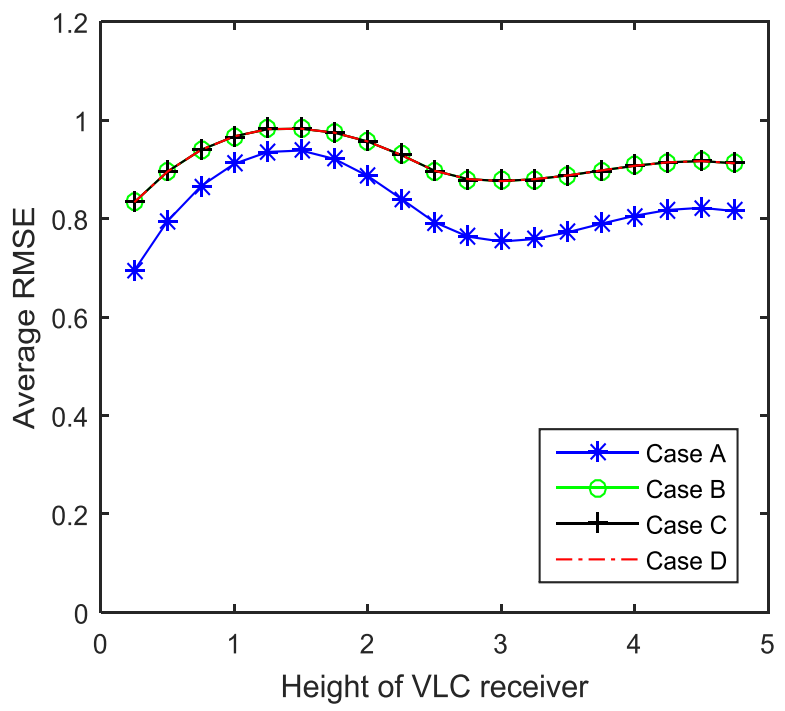

Fig. 6 Effect of VLC height in whole places of room.

As depicted in Figure 6, the height of VLC receiver does not have considerable effect on the results and error is almost fixed. As we expected in case A, where we have 4 VAPs, that error would be less than other cases. Also, when we use two VAPs, error is acceptable. Additionally, simulation result confirms that in case D where we have 4 VAPs with simpler design, error is almost the same as what it is in case A. 
C. Effect of Number of LEDs

In the third scenario, we investigate the effect of number of LEDs in VAPs. In this simulation, we vary the number of LEDs in VAPs and measure the error in each case. We move the receiver in whole places of room in the height of 2.5 meter and calculate the average of error. Results are depicted in Figure 7.

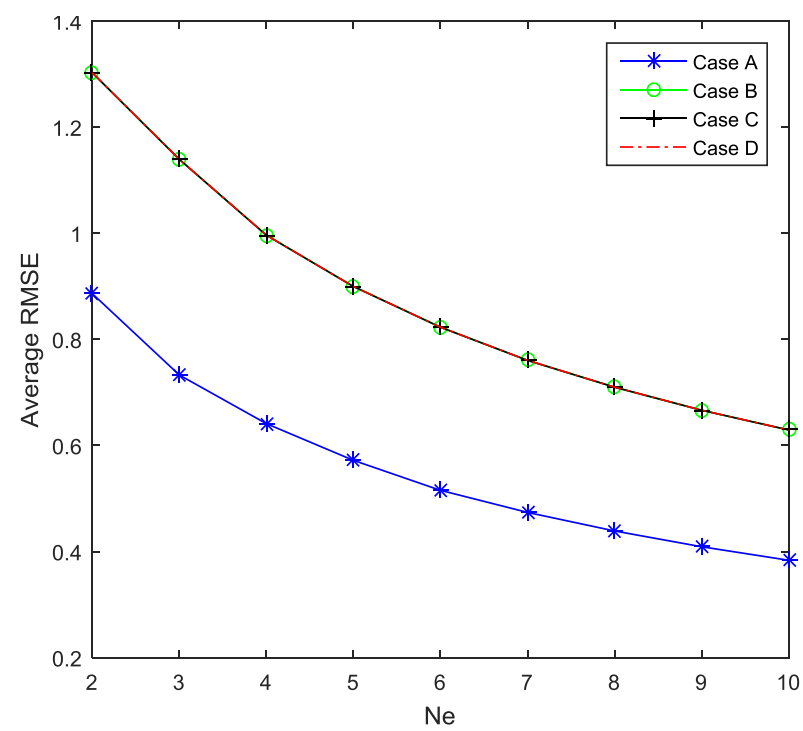

Fig. 7 Effect of number of LEDs.

As depicted in Figure 7, by increasing the number of LEDs in VAPs, localization error becomes less. Also, results show that by using two VAPs, we can localize VLC receiver with about 0.6 meter error.

\section{CONCLUSION}

According to the importance of localization and wireless spectrum scarcity, we introduce indoor localization using VLC. In this paper we use connection information to localize a VLC receiver in 3D indoor places. Simulation results confirm that we can localize a VLC receiver using 2 VAPs. Also simulation results show that if we use VAPs which can measure azimuthal or polar angles, we can localize VLC receiver by an acceptable error and if we use 4 VAPs with azimuthal and polar angles, localization error becomes less than 0.4 meter.

\section{REFERENCES}

[1] A. Sevincer, A. Bhattarai, M. Bilgi, M. Yuksel, and N. Pala, "LIGHTNETs: Smart LIGHTing and mobile optical wireless NETworks-A survey," IEEE Communications Surveys \& Tutorials, vol. 15, pp. 1620-1641, 2013.

[2] C. A. M, x, ilean, M. Dimian, V. Popa, L. Chassagne, et al., "Novel DSP Receiver Architecture for Multi-Channel Visible Light Communications in Automotive Applications," IEEE Sensors Journal, vol. 16, pp. 3597-3602, 2016.

[3] S. Pergoloni, M. Biagi, S. Colonnese, R. Cusani, and G. Scarano, "Optimized LEDs Footprinting for Indoor Visible Light Communication Networks," IEEE Photonics Technology Letters, vol. 28, pp. 532-535, 2016.

[4] Y. Yang, Z. Zeng, J. Cheng, and C. Guo, "An Enhanced DCOOFDM Scheme for Dimming Control in Visible Light Communication Systems," IEEE Photonics Journal, vol. 8, pp. 113,2016
[5] Y. S. Erogluy, I. Guvency, N. Palay, and M. Yukselz, "AOA-based localization and tracking in multi-element VLC systems," in Wireless and Microwave Technology Conference (WAMICON), IEEE 16th Annual, pp. 1-5, 2015.

[6] A. H. Sayed, A. Tarighat, and N. Khajehnouri, "Network-based wireless location: challenges faced in developing techniques for accurate wireless location information," IEEE signal processing magazine, vol. 22, pp. 24-40, 2005.

[7] S.-Y. Jung, S. Hann, and C.-S. Park, "TDOA-based optical wireless indoor localization using LED ceiling lamps," IEEE Transactions on Consumer Electronics, vol. 57, pp. 1592-1597, 2011.

[8] J. Nah, R. Parthiban, and M. H. Jaward, "Visible Light Communications localization using TDOA-based coherent heterodyne detection," in IEEE 4th International Conference on Photonics (ICP), pp. 247-249, 2013

[9] N.-T. Nguyen, A. Suebsomran, K. Sripimanwat, and N.-H. Nguyen, "Design and simulation of a novel indoor mobile robot localization method using a light-emitting diode positioning system," Transactions of the Institute of Measurement and Control, vol. 38, pp. 305-314, 2016. 